\title{
An explicit algorithm for solving the optimize hierarchical problems
}

Poom Kumam ${ }^{1,2}$, Thanyarat Jitpeera ${ }^{3 *}$ and Wararat Yarangkham ${ }^{4}$

${ }^{*}$ Correspondence:

t.jitpeera@hotmail.com

${ }^{3}$ Department of Mathematics, Faculty of Science and Agriculture, Rajamangala University of Technology Lanna, Phan, Chiangrai, 57120, Thailand

Full list of author information is available at the end of the article

\begin{abstract}
In this paper, we consider the variational inequality problem over the generalized mixed equilibrium problem which has a hierarchical structure. Strong convergence of the algorithm to the unique solution is guaranteed under some assumptions.

MSC: 47H09; 47H10; 47J20; 49J40; 65J15

Keywords: nonexpansive; strong convergence; variational inequality; fixed point; hierarchical problem
\end{abstract}

\section{Introduction}

Let $C$ be a closed convex subset of a real Hilbert space $H$ with the inner product $\langle\cdot, \cdot\rangle$ and the norm $\|\cdot\|$. We denote weak convergence and strong convergence by the notations $\rightarrow$ and $\rightarrow$, respectively. Let $A: C \rightarrow H$ be a nonlinear mapping and let $F$ be a bifunction of $C \times C$ into $\mathcal{R}$, where $\mathcal{R}$ is the set of real numbers.

Consider the generalized mixed equilibrium problem which is to find $x \in C$ such that

$$
F(x, y)+\langle A x, y-x\rangle+\varphi(y)-\varphi(x) \geq 0, \quad \forall y \in C .
$$

The solution set of (1.1) is denoted by $\operatorname{GMEP}(F, \varphi, A)$. See [1-4].

If $\varphi \equiv 0$, the problem (1.1) is reduced to the generalized equilibrium problem which is to find $x \in C$ such that

$$
F(x, y)+\langle A x, y-x\rangle \geq 0, \quad \forall y \in C .
$$

The set of solutions of (1.2) is denoted by $\operatorname{GEP}(F, A)$.

If $A \equiv 0$ and $\varphi \equiv 0$, the problem (1.1) is reduced to the equilibrium problem [5] which is to find $x \in C$ such that

$$
F(x, y) \geq 0, \quad \forall y \in C .
$$

The solution set of (1.3) is denoted by $E P(F)$.

If $F \equiv 0$ and $\varphi \equiv 0$, the problem (1.1) is reduced to the Hartmann-Stampacchia variational inequality [6] which is to find $x \in C$ such that

$$
\langle A x, y-x\rangle \geq 0, \quad \forall y \in C .
$$

The solution set of (1.4) is denoted by $V I(C, A)$.

\section{Springer}

(02014 Kumam et al.; licensee Springer. This is an Open Access article distributed under the terms of the Creative Commons Attribution License (http://creativecommons.org/licenses/by/2.0), which permits unrestricted use, distribution, and reproduction in any medium, provided the original work is properly cited. 
A mapping $T: C \rightarrow C$ is called nonexpansive if $\|T x-T y\| \leq\|x-y\|$ for all $x, y \in C$. If $C$ is bounded closed convex and $T$ is a nonexpansive mapping of $C$ into itself, then $F(T)$ is nonempty [7]. A point $x \in H$ is a fixed point of $T$ provided $T x=x$. Denote by $F(T)$ the set of fixed points of $T$; that is, $F(T)=\{x \in H: T x=x\}$.

We discuss the following variational inequality problem over the generalized mixed equilibrium problem, which is called the hierarchical problem over the generalized mixed equilibrium problem, which is to find a point $x \in \operatorname{GMEP}(F, \varphi, B)$ such that

$$
\langle A x, y-x\rangle \geq 0, \quad \forall y \in \operatorname{GMEP}(F, \varphi, B)
$$

where $A, B$ are two monotone operators. See $[8,9]$.

A mapping $A: C \rightarrow C$ is called $\alpha$-strongly monotone if there exists a positive real number $\alpha$ such that $\langle A x-A y, x-y\rangle \geq \alpha\|x-y\|^{2}$ for all $x, y \in C$. A mapping $A: C \rightarrow C$ is called $L-$ Lipschitz continuous if there exists a positive real number $L$ such that $\|A x-A y\| \leq L\|x-y\|$ for all $x, y \in C$. A linear bounded operator $A$ is called strongly positive on $H$ if there exists a constant $\bar{\gamma}>0$ with the property $\langle A x, x\rangle \geq \bar{\gamma}\|x\|^{2}$ for all $x \in H$. A mapping $f: C \rightarrow H$ is called a $\rho$-contraction if there exists a constant $\rho \in[0,1)$ such that $\|f(x)-f(y)\| \leq \rho\|x-y\|$ for all $x, y \in C$.

In 2010, Yao et al. [10] considered the hierarchical problem over the generalized equilibrium problem, $x_{s, t}$ being defined by implicit algorithms:

$$
x_{s, t}=s\left[t f\left(x_{s, t}\right)+(1-t)\left(x_{s, t}-\lambda A x_{s, t}\right)\right]+(1-s) T_{r}\left(x_{s, t}-r B x_{s, t}\right), \quad s, t \in(0,1),
$$

for each $(s, t) \in(0,1)^{2}$. The net $x_{s, t}$ hierarchically converges to the unique solution $x^{*}$ of the problem of the variational inequality which is to find a point $x^{*} \in \operatorname{GEP}(F, B)$ such that

$$
\left\langle A x^{*}, x-x^{*}\right\rangle \geq 0, \quad \forall x \in G E P(F, B)
$$

where $A, B$ are two monotone operators. The solution set of (1.6) is denoted by $\Omega$. Furthermore, $x^{*}$ also solves the following variational inequality:

$$
x^{*} \in \Omega, \quad\left\langle(I-f) x^{*}, x-x^{*}\right\rangle \geq 0, \quad \forall x \in \Omega .
$$

In 2011, Yao et al. [11] studied the hierarchical problem over the fixed point set. Let the sequence $\left\{x_{n}\right\}$ be generated by two algorithms as follows.

Implicit Algorithm: $x_{t}=T P_{C}[I-t(A-\gamma f)] x_{t}, \forall t \in(0,1)$ and

Explicit Algorithm: $x_{n+1}=\beta_{n} x_{n}+\left(1-\beta_{n}\right) T P_{C}\left[I-\alpha_{n}(A-\gamma f)\right] x_{n}, \forall n \geq 0$.

They showed that these two algorithms converge strongly to the unique solution of the problem of the variational inequality which is to find $x^{*} \in F(T)$ such that

$$
\left\langle(A-\gamma f) x^{*}, x-x^{*}\right\rangle \geq 0, \quad \forall x \in F(T),
$$

where $A: C \rightarrow H$ is a strongly positive linear bounded operator, $f: C \rightarrow H$ is a $\rho$ contraction, and $T: C \rightarrow C$ is a nonexpansive mapping.

In this paper, we construct an algorithm and introduce the hierarchical problem over the generalized mixed equilibrium problem. The sequence $\left\{x_{n}\right\}$ is generated by the algorithm 
for $x_{0} \in C$,

$$
x_{n+1}=\alpha_{n}\left(\beta_{n} x_{n}+\left(1-\beta_{n}\right) P_{C}\left[I-\lambda_{n}(A-\gamma f)\right] x_{n}\right)+\left(1-\alpha_{n}\right) T_{r_{n}}\left(I-r_{n} B\right) x_{n},
$$

where $\left\{\alpha_{n}\right\},\left\{\beta_{n}\right\},\left\{\lambda_{n}\right\} \subset[0,1]$, and $r_{n} \in(0,2 \beta)$ satisfy some conditions. Then $\left\{x_{n}\right\}$ converges strongly to $x^{*} \in \operatorname{GMEP}(F, \varphi, B)$, which is the unique solution of the variational inequality:

$$
\left\langle(A-\gamma f) x^{*}, x-x^{*}\right\rangle \geq 0, \quad \forall x \in G M E P(F, \varphi, B) .
$$

Our results improve the results of Yao et al. [10], Yao et al. [11] and some other authors.

\section{Preliminaries}

Let $C$ be a nonempty closed convex subset of $H$. We have the following inequality in an inner product space: $\|x+y\|^{2} \leq\|x\|^{2}+2\langle y, x+y\rangle, \forall x, y \in H$. For every point $x \in H$, there exists a unique nearest point in $C$, denoted by $P_{C} x$, such that

$$
\left\|x-P_{C} x\right\| \leq\|x-y\|, \quad \text { for all } y \in C .
$$

$P_{C}$ is called the metric projection of $H$ onto $C$. It is well known that $P_{C}$ is a nonexpansive mapping of $H$ onto $C$ and satisfies

$$
\left\langle x-y, P_{C} x-P_{C} y\right\rangle \geq\left\|P_{C} x-P_{C} y\right\|^{2}
$$

for every $x, y \in H$. Moreover, $P_{C} x$ is characterized by the following properties: $P_{C} x \in C$ and

$$
\begin{aligned}
& \left\langle x-P_{C} x, y-P_{C} x\right\rangle \leq 0, \\
& \|x-y\|^{2} \geq\left\|x-P_{C} x\right\|^{2}+\left\|y-P_{C} x\right\|^{2},
\end{aligned}
$$

for all $x \in H, y \in C$. Let $B$ be a monotone mapping of $C$ into $H$. In the context of the variational inequality problem the characterization of projection (2.1) implies the following:

$$
u \in V I(C, B) \Leftrightarrow u=P_{C}(u-\lambda B u), \quad \lambda>0 .
$$

It is also well known that $H$ satisfies the Opial condition [12], i.e., for any sequence $\left\{x_{n}\right\} \subset H$ with $x_{n} \rightarrow x$, the inequality $\liminf _{n \rightarrow \infty}\left\|x_{n}-x\right\|<\liminf _{n \rightarrow \infty}\left\|x_{n}-y\right\|$, holds for every $y \in H$ with $x \neq y$.

For solving the generalized mixed equilibrium problem and the mixed equilibrium problem, let us give the following assumptions for the bifunction $F, \varphi$, and the set $C$ :

(A1) $F(x, x)=0$ for all $x \in C$;

(A2) $F$ is monotone, i.e., $F(x, y)+F(y, x) \leq 0$ for all $x, y \in C$;

(A3) for each $y \in C, x \mapsto F(x, y)$ is weakly upper semicontinuous;

(A4) for each $x \in C, y \mapsto F(x, y)$ is convex;

(A5) for each $x \in C, y \mapsto F(x, y)$ is lower semicontinuous; 
(B1) for each $x \in H$ and $r>0$, there exist a bounded subset $D_{x} \subseteq C$ and $y_{x} \in C$ such that for any $z \in C \backslash D_{x}$,

$$
F\left(z, y_{x}\right)+\varphi\left(y_{x}\right)-\varphi(z)+\frac{1}{r}\left\langle y_{x}-z, z-x\right\rangle<0
$$

(B2) $C$ is a bounded set.

Lemma 2.1 [13] Let $C$ be a nonempty closed convex subset of a real Hilbert space H. Let $F$ be a bifunction from $C \times C$ to $\mathcal{R}$ satisfying (A1)-(A5) and let $\varphi: C \rightarrow \mathcal{R}$ be a proper lower semicontinuous and convex function. For $r>0$ and $x \in H$, define a mapping $T_{r}: H \rightarrow C$ as follows.

$$
T_{r}(x)=\left\{z \in C: F(z, y)+\varphi(y)-\varphi(z)+\frac{1}{r}\langle y-z, z-x\rangle \geq 0, \forall y \in C\right\}
$$

for all $x \in H$. Assume that either (B1) or (B2) holds. Then the following results hold:

(1) for each $x \in H, T_{r}(x) \neq \emptyset$;

(2) $T_{r}$ is single-valued;

(3) $T_{r}$ is firmly nonexpansive, i.e., for any $x, y \in H$, $\left\|T_{r} x-T_{r} y\right\|^{2} \leq\left\langle T_{r} x-T_{r} y, x-y\right\rangle$;

(4) $F\left(T_{r}\right)=\operatorname{MEP}(F, \varphi)$

(5) $\operatorname{MEP}(F, \varphi)$ is closed and convex.

Lemma 2.2 [14] Let C be a closed convex subset of a real Hilbert space H and let $T: C \rightarrow C$ be a nonexpansive mapping. Then $I-T$ is demiclosed at zero, that is, $x_{n} \rightarrow x, x_{n}-T x_{n} \rightarrow 0$ implies $x=T x$.

Lemma 2.3 [15] Assume $A$ is a self adjoint and strongly positive linear bounded operator on a Hilbert space $H$ with coefficient $\bar{\gamma}>0$ and $0<\rho \leq\|A\|^{-1}$, then $\|I-\rho A\| \leq 1-\rho \bar{\gamma}$.

Lemma $2.4[16]$ Assume $\left\{a_{n}\right\}$ is a sequence of nonnegative real numbers such that

$$
a_{n+1} \leq\left(1-\gamma_{n}\right) a_{n}+\delta_{n}, \quad \forall n \geq 0,
$$

where $\left\{\gamma_{n}\right\} \subset(0,1)$ and $\left\{\delta_{n}\right\}$ are sequences in $\mathcal{R}$ such that

(i) $\sum_{n=1}^{\infty} \gamma_{n}=\infty$,

(ii) $\limsup _{n \rightarrow \infty} \frac{\delta_{n}}{\gamma_{n}} \leq 0$ or $\sum_{n=1}^{\infty}\left|\delta_{n}\right|<\infty$.

Then $\lim _{n \rightarrow \infty} a_{n}=0$.

\section{Strong convergence theorems}

In this section, we introduce an explicit algorithm for solving some hierarchical problem over the set of fixed points of a nonexpansive and the generalized mixed equilibrium problem.

Theorem 3.1 Let $C$ be a nonempty closed convex subset of a real Hilbert space $H, A$ : $H \rightarrow H$ be a strongly positive linear bounded operator, $f: C \rightarrow H$ be $\rho$-contraction, $\gamma$ be a positive real number such that $\frac{\bar{\gamma}-1}{\rho}<\gamma<\frac{\bar{\gamma}}{\rho}$. Let $B: C \rightarrow H$ be $\beta$-inverse-strongly monotone and $F$ be a bifunction from $C \times C \rightarrow \mathcal{R}$ satisfying (A1)-(A5) and let $\varphi: C \rightarrow \mathcal{R}$ be convex 
and lower semicontinuous with either (B1) or (B2). Let $\left\{x_{n}\right\}$ be a sequence generated by the following algorithm for arbitrary $x_{0} \in C$ :

$$
x_{n+1}=\alpha_{n}\left(\beta_{n} x_{n}+\left(1-\beta_{n}\right) P_{C}\left[I-\lambda_{n}(A-\gamma f)\right] x_{n}\right)+\left(1-\alpha_{n}\right) T_{r_{n}}\left(I-r_{n} B\right) x_{n},
$$

where $\left\{\alpha_{n}\right\},\left\{\beta_{n}\right\},\left\{\lambda_{n}\right\} \subset[0,1], \alpha_{n} \leq \lambda_{n}$, and $r_{n} \in(0,2 \beta)$ satisfy the following conditions:

(C1) $\sum_{n=1}^{\infty}\left|\alpha_{n}-\alpha_{n-1}\right|<\infty$;

(C2) $\sum_{n=1}^{\infty}\left|\beta_{n}-\beta_{n-1}\right|<\infty$;

(C3) $\sum_{n=1}^{\infty}\left|\lambda_{n}-\lambda_{n-1}\right|<\infty, \sum_{n=1}^{\infty} \lambda_{n}=\infty, \lim _{n \rightarrow \infty} \lambda_{n}=0$;

(C4) $\sum_{n=1}^{\infty}\left|r_{n}-r_{n-1}\right|<\infty, \liminf _{n \rightarrow \infty} r_{n}>0$.

Then $\left\{x_{n}\right\}$ converges strongly to $x^{*} \in \operatorname{GMEP}(F, \varphi, B)$, which is the unique solution of the variational inequality:

$$
\left\langle(A-\gamma f) x^{*}, x-x^{*}\right\rangle \geq 0, \quad \forall x \in \operatorname{GMEP}(F, \varphi, B) .
$$

Proof We will divide the proof into five steps.

Step 1. We will show $\left\{x_{n}\right\}$ is bounded. For any $q \in \operatorname{GMEP}(F, \varphi, B)$ and taking $y_{n}=P_{C}[I-$ $\left.\lambda_{n}(A-\gamma f)\right] x_{n}$, we note that

$$
\begin{aligned}
\left\|y_{n}-q\right\| & =\left\|P_{C}\left[I-\lambda_{n}(A-\gamma f)\right] x_{n}-P_{C} q\right\| \\
& \leq\left\|\left[I-\lambda_{n}(A-\gamma f)\right] x_{n}-q\right\| \\
& \leq \lambda_{n}\left\|\gamma f\left(x_{n}\right)-\gamma f(q)\right\|+\lambda_{n}\|\gamma f(q)-A q\|+\left|I-\lambda_{n} A\right|\left\|x_{n}-q\right\| \\
& \leq \lambda_{n} \gamma \rho\left\|x_{n}-q\right\|+\lambda_{n}\|\gamma f(q)-A q\|+\left(1-\lambda_{n} \bar{\gamma}\right)\left\|x_{n}-q\right\| \\
& =\left[1-(\bar{\gamma}-\gamma \rho) \lambda_{n}\right]\left\|x_{n}-q\right\|+\lambda_{n}\|\gamma f(q)-A q\| .
\end{aligned}
$$

From (3.1), we have

$$
\begin{aligned}
\left\|x_{n+1}-q\right\|= & \left\|\alpha_{n}\left\{\beta_{n} x_{n}+\left(1-\beta_{n}\right) y_{n}\right\}+\left(1-\alpha_{n}\right) T_{r_{n}}\left(I-r_{n} B\right) x_{n}-q\right\| \\
\leq & \alpha_{n}\left\|\beta_{n} x_{n}+\left(1-\beta_{n}\right) y_{n}-q\right\|+\left(1-\alpha_{n}\right)\left\|T_{r_{n}}\left(I-r_{n} B\right) x_{n}-q\right\| \\
\leq & \alpha_{n} \beta_{n}\left\|x_{n}-q\right\|+\alpha_{n}\left(1-\beta_{n}\right)\left\|y_{n}-q\right\| \\
& +\left(1-\alpha_{n}\right)\left\|T_{r_{n}}\left(I-r_{n} B\right) x_{n}-T_{r_{n}}\left(I-r_{n} B\right) q\right\| \\
\leq & \alpha_{n} \beta_{n}\left\|x_{n}-q\right\|+\alpha_{n}\left(1-\beta_{n}\right)\left\{\left[1-(\bar{\gamma}-\gamma \rho) \lambda_{n}\right]\left\|x_{n}-q\right\|+\lambda_{n}\|\gamma f(q)-A q\|\right\} \\
& +\left(1-\alpha_{n}\right)\left\|x_{n}-q\right\| \\
= & \alpha_{n} \beta_{n}\left\|x_{n}-q\right\|+\alpha_{n}\left(1-\beta_{n}\right)\left[1-(\bar{\gamma}-\gamma \rho) \lambda_{n}\right]\left\|x_{n}-q\right\| \\
& +\alpha_{n}\left(1-\beta_{n}\right) \lambda_{n}\|\gamma f(q)-A q\|+\left(1-\alpha_{n}\right)\left\|x_{n}-q\right\| \\
= & {\left[1-\alpha_{n}\left(1-\beta_{n}\right)\right]\left\|x_{n}-q\right\|+\alpha_{n}\left(1-\beta_{n}\right)\left[1-(\bar{\gamma}-\gamma \rho) \lambda_{n}\right]\left\|x_{n}-q\right\| } \\
& +\alpha_{n}\left(1-\beta_{n}\right) \lambda_{n}\|\gamma f(q)-A q\| \\
= & \left\{1-\alpha_{n}\left(1-\beta_{n}\right)\left(1-\left[1-(\bar{\gamma}-\gamma \rho) \lambda_{n}\right]\right)\right\}\left\|x_{n}-q\right\| \\
& +\alpha_{n}\left(1-\beta_{n}\right) \lambda_{n}\|\gamma f(q)-A q\|
\end{aligned}
$$




$$
\begin{aligned}
= & {\left[1-\alpha_{n}\left(1-\beta_{n}\right) \lambda_{n}(\bar{\gamma}-\gamma \rho)\right]\left\|x_{n}-q\right\|+\alpha_{n}\left(1-\beta_{n}\right) \lambda_{n}\|\gamma f(q)-A q\| } \\
= & {\left[1-\alpha_{n}\left(1-\beta_{n}\right) \lambda_{n}(\bar{\gamma}-\gamma \rho)\right]\left\|x_{n}-q\right\| } \\
& +\alpha_{n}\left(1-\beta_{n}\right) \lambda_{n}(\bar{\gamma}-\gamma \rho) \frac{\|\gamma f(q)-A q\|}{\bar{\gamma}-\gamma \rho} .
\end{aligned}
$$

It follows by induction that

$$
\left\|x_{n}-q\right\| \leq \max \left\{\left\|x_{0}-q\right\|, \frac{\|\gamma f(q)-A q\|}{\bar{\gamma}-\gamma \rho}\right\}, \quad \forall n \geq 0 .
$$

Therefore $\left\{x_{n}\right\}$ is bounded and so are $\left\{y_{n}\right\},\left\{A x_{n}\right\}$, and $\left\{f\left(x_{n}\right)\right\}$.

Step 2. We show that $\lim _{n \rightarrow \infty}\left\|x_{n+1}-x_{n}\right\|=0$. Setting $v_{n}=\left[I-\lambda_{n}(A-\gamma f)\right] x_{n}$ and we observe that

$$
\begin{aligned}
\left\|y_{n+1}-y_{n}\right\|= & \left\|P_{C} v_{n+1}-P_{C} v_{n}\right\| \\
\leq & \left\|\left[I-\lambda_{n+1}(A-\gamma f)\right] x_{n+1}-\left[I-\lambda_{n}(A-\gamma f)\right] x_{n}\right\| \\
= & \| \lambda_{n+1} \gamma\left[f\left(x_{n+1}\right)-f\left(x_{n}\right)\right]+\left(\lambda_{n+1}-\lambda_{n}\right) \gamma f\left(x_{n}\right)+\left(I-\lambda_{n+1} A\right)\left(x_{n+1}-x_{n}\right) \\
& +\left(\lambda_{n+1}-\lambda_{n}\right) A x_{n} \| \\
\leq & \lambda_{n+1} \gamma\left\|f\left(x_{n+1}\right)-f\left(x_{n}\right)\right\|+\left(1-\lambda_{n+1} \bar{\gamma}\right)\left\|x_{n+1}-x_{n}\right\| \\
& +\left|\lambda_{n+1}-\lambda_{n}\right|\left(\left\|\gamma f\left(x_{n}\right)\right\|+\left\|A x_{n}\right\|\right) \\
\leq & \lambda_{n+1} \gamma \rho\left\|x_{n+1}-x_{n}\right\|+\left(1-\lambda_{n+1} \bar{\gamma}\right)\left\|x_{n+1}-x_{n}\right\| \\
& +\left|\lambda_{n+1}-\lambda_{n}\right|\left(\left\|\gamma f\left(x_{n}\right)\right\|+\left\|A x_{n}\right\|\right) \\
= & {\left[1-(\bar{\gamma}-\gamma \rho) \lambda_{n+1}\right]\left\|x_{n+1}-x_{n}\right\|+\left|\lambda_{n+1}-\lambda_{n}\right| M_{1} }
\end{aligned}
$$

where $M_{1}=\sup \left\{\left\|\gamma f\left(x_{n}\right)\right\|+\left\|A x_{n}\right\|: n \in \mathbb{N}\right\}$. Setting $z_{n}=\beta_{n} x_{n}+\left(1-\beta_{n}\right) y_{n}$ for all $n \geq 0$. We observes that

$$
\begin{aligned}
\left\|z_{n+1}-z_{n}\right\| & =\left\|\beta_{n+1} x_{n+1}+\left(1-\beta_{n+1}\right) y_{n+1}-\left(\beta_{n} x_{n}+\left(1-\beta_{n}\right) y_{n}\right)\right\| \\
& \leq \beta_{n+1}\left\|x_{n+1}-x_{n}\right\|+\left|\beta_{n+1}-\beta_{n}\right|\left\|x_{n}-y_{n}\right\|+\left|1-\beta_{n+1}\right|\left\|y_{n+1}-y_{n}\right\| .
\end{aligned}
$$

Substituting (3.4) into (3.5) it follows that

$$
\begin{aligned}
\left\|z_{n+1}-z_{n}\right\| \leq & \beta_{n+1}\left\|x_{n+1}-x_{n}\right\|+\left|\beta_{n+1}-\beta_{n}\right|\left\|x_{n}-y_{n}\right\| \\
& +\left|1-\beta_{n+1}\right|\left\{\left[1-(\bar{\gamma}-\gamma \rho) \lambda_{n+1}\right]\left\|x_{n+1}-x_{n}\right\|+\left|\lambda_{n+1}-\lambda_{n}\right| M_{1}\right\} \\
\leq & \beta_{n+1}\left\|x_{n+1}-x_{n}\right\|+\left|\beta_{n+1}-\beta_{n}\right|\left\|x_{n}-y_{n}\right\| \\
& +\left[1-\beta_{n+1}-\left(1-\beta_{n+1}\right)(\bar{\gamma}-\gamma \rho) \lambda_{n+1}\right]\left\|x_{n+1}-x_{n}\right\|+\left|\lambda_{n+1}-\lambda_{n}\right| M_{1} \\
= & {\left[1-\left(1-\beta_{n+1}\right)(\bar{\gamma}-\gamma \rho) \lambda_{n+1}\right]\left\|x_{n+1}-x_{n}\right\|+\left|\beta_{n+1}-\beta_{n}\right| M_{2} } \\
& +\left|\lambda_{n+1}-\lambda_{n}\right| M_{1},
\end{aligned}
$$


where $M_{2}=\sup \left\{\left\|x_{n}-y_{n}\right\|: n \in \mathbb{N}\right\}$. On the other hand, from $u_{n-1}=T_{r_{n-1}}\left(x_{n-1}-r_{n-1} B x_{n-1}\right)$ and $u_{n}=T_{r_{n}}\left(x_{n}-r_{n} B x_{n}\right)$ it follows that

$$
\begin{aligned}
& F\left(u_{n-1}, y\right)+\left\langle B x_{n-1}, y-u_{n-1}\right\rangle+\varphi(y)-\varphi\left(u_{n-1}\right)+\frac{1}{r_{n-1}}\left\langle y-u_{n-1}, u_{n-1}-x_{n-1}\right\rangle \geq 0, \\
& \quad \forall y \in C
\end{aligned}
$$

and

$$
F\left(u_{n}, y\right)+\left\langle B x_{n}, y-u_{n}\right\rangle+\varphi(y)-\varphi\left(u_{n}\right)+\frac{1}{r_{n}}\left\langle y-u_{n}, u_{n}-x_{n}\right\rangle \geq 0, \quad \forall y \in C .
$$

Substituting $y=u_{n}$ into (3.7) and $y=u_{n-1}$ into (3.8), we have

$$
F\left(u_{n-1}, u_{n}\right)+\left\langle B x_{n-1}, u_{n}-u_{n-1}\right\rangle+\varphi\left(u_{n}\right)-\varphi\left(u_{n-1}\right)+\frac{1}{r_{n-1}}\left\langle u_{n}-u_{n-1}, u_{n-1}-x_{n-1}\right\rangle \geq 0
$$

and

$$
F\left(u_{n}, u_{n-1}\right)+\left\langle B x_{n}, u_{n-1}-u_{n}\right\rangle+\varphi\left(u_{n-1}\right)-\varphi\left(u_{n}\right)+\frac{1}{r_{n}}\left\langle u_{n-1}-u_{n}, u_{n}-x_{n}\right\rangle \geq 0 .
$$

From (A2), we have

$$
\left\langle u_{n}-u_{n-1}, B x_{n-1}-B x_{n}+\frac{u_{n-1}-x_{n-1}}{r_{n-1}}-\frac{u_{n}-x_{n}}{r_{n}}\right\rangle \geq 0,
$$

and then

$$
\left\langle u_{n}-u_{n-1}, r_{n-1}\left(B x_{n-1}-B x_{n}\right)+u_{n-1}-x_{n-1}-\frac{r_{n-1}}{r_{n}}\left(u_{n}-x_{n}\right)\right\rangle \geq 0,
$$

so

$$
\left\langle u_{n}-u_{n-1}, r_{n-1} B x_{n-1}-r_{n-1} B x_{n}+u_{n-1}-u_{n}+u_{n}-x_{n-1}-\frac{r_{n-1}}{r_{n}}\left(u_{n}-x_{n}\right)\right\rangle \geq 0 .
$$

It follows that

$$
\begin{aligned}
& \left\langle u_{n}-u_{n-1},\left(I-r_{n-1} B\right) x_{n}-\left(I-r_{n-1} B\right) x_{n-1}+u_{n-1}-u_{n}+u_{n}-x_{n}-\frac{r_{n-1}}{r_{n}}\left(u_{n}-x_{n}\right)\right\rangle \geq 0, \\
& \left\langle u_{n}-u_{n-1}, u_{n-1}-u_{n}\right\rangle+\left\langle u_{n}-u_{n-1}, x_{n}-x_{n-1}+\left(1-\frac{r_{n-1}}{r_{n}}\right)\left(u_{n}-x_{n}\right)\right\rangle \geq 0 .
\end{aligned}
$$

Without loss of generality, let us assume that there exists a real number $c$ such that $r_{n-1}>$ $c>0$, for all $n \in \mathbb{N}$. Then we have

$$
\begin{aligned}
\left\|u_{n}-u_{n-1}\right\|^{2} & \leq\left\langle u_{n}-u_{n-1}, x_{n}-x_{n-1}+\left(1-\frac{r_{n-1}}{r_{n}}\right)\left(u_{n}-x_{n}\right)\right\rangle \\
& \leq\left\|u_{n}-u_{n-1}\right\|\left\{\left\|x_{n}-x_{n-1}\right\|+\left|1-\frac{r_{n-1}}{r_{n}}\right|\left\|u_{n}-x_{n}\right\|\right\}
\end{aligned}
$$


and hence

$$
\begin{aligned}
\left\|u_{n}-u_{n-1}\right\| & \leq\left\|x_{n}-x_{n-1}\right\|+\frac{1}{r_{n}}\left|r_{n}-r_{n-1}\right|\left\|u_{n}-x_{n}\right\| \\
& \leq\left\|x_{n}-x_{n-1}\right\|+\frac{M_{3}}{c}\left|r_{n}-r_{n-1}\right|,
\end{aligned}
$$

where $M_{3}=\sup \left\{\left\|u_{n}-x_{n}\right\|: n \in \mathbb{N}\right\}$. From (3.1), we have

$$
\begin{aligned}
\left\|x_{n+1}-x_{n}\right\| & =\left\|\alpha_{n} z_{n}+\left(1-\alpha_{n}\right) u_{n}-\alpha_{n-1} z_{n-1}-\left(1-\alpha_{n-1}\right) u_{n-1}\right\| \\
& \leq \alpha_{n}\left\|z_{n}-z_{n-1}\right\|+\left|\alpha_{n}-\alpha_{n-1}\right|\left\|z_{n-1}-u_{n-1}\right\|+\left|1-\alpha_{n}\right|\left\|u_{n}-u_{n-1}\right\| \\
& =\alpha_{n}\left\|z_{n}-z_{n-1}\right\|+\left|\alpha_{n}-\alpha_{n-1}\right| M_{4}+\left|1-\alpha_{n}\right|\left\|u_{n}-u_{n-1}\right\|,
\end{aligned}
$$

where $M_{4}=\sup \left\{\left\|z_{n}-u_{n}\right\|: n \in \mathbb{N}\right\}$. Substituting (3.6) and (3.9) into (3.10)

$$
\begin{aligned}
\left\|x_{n+1}-x_{n}\right\| \leq & \alpha_{n}\left\{\left[1-\left(1-\beta_{n}\right)(\bar{\gamma}-\gamma \rho) \lambda_{n}\right]\left\|x_{n}-x_{n-1}\right\|\right. \\
& \left.+\left|\beta_{n}-\beta_{n-1}\right| M_{2}+\left|\lambda_{n}-\lambda_{n-1}\right| M_{1}\right\} \\
& +\left|\alpha_{n}-\alpha_{n-1}\right| M_{4}+\left|1-\alpha_{n}\right|\left\{\left\|x_{n}-x_{n-1}\right\|+\frac{M_{3}}{c}\left|r_{n}-r_{n-1}\right|\right\} \\
\leq & {\left[1-\left(1-\beta_{n}\right)(\bar{\gamma}-\gamma \rho) \alpha_{n} \lambda_{n}\right]\left\|x_{n}-x_{n-1}\right\|+\alpha_{n}\left|\beta_{n}-\beta_{n-1}\right| M_{2} } \\
& +\alpha_{n}\left|\lambda_{n}-\lambda_{n-1}\right| M_{1}+\left|\alpha_{n}-\alpha_{n-1}\right| M_{4}+\frac{M_{3}}{c}\left|r_{n}-r_{n-1}\right|,
\end{aligned}
$$

from (C1)-(C4) and the boundedness of $\left\{x_{n}\right\},\left\{y_{n}\right\},\left\{z_{n}\right\},\left\{f\left(x_{n}\right)\right\}$, and $\left\{A x_{n}\right\}$. Applying Lemma 2.4, we obtain

$$
\lim _{n \rightarrow \infty}\left\|x_{n+1}-x_{n}\right\|=0 .
$$

Step 3. We show that $\lim _{n \rightarrow \infty}\left\|x_{n}-u_{n}\right\|=0$. For each $q \in \operatorname{GMEP}(F, \varphi, B)$, note that $T_{r_{n}}$ is firmly nonexpansive, then we have

$$
\begin{aligned}
\left\|u_{n}-q\right\|^{2}= & \left\|T_{r_{n}}\left(x_{n}-r_{n} B x_{n}\right)-T_{r_{n}}\left(q-r_{n} B q\right)\right\|^{2} \\
\leq & \left\langle T_{r_{n}}\left(x_{n}-r_{n} B x_{n}\right)-T_{r_{n}}\left(q-r_{n} B q\right), u_{n}-q\right\rangle \\
= & \left\langle\left(x_{n}-r_{n} B x_{n}\right)-\left(q-r_{n} B q\right), u_{n}-q\right\rangle \\
= & \frac{1}{2}\left\{\left\|\left(x_{n}-r_{n} B x_{n}\right)-\left(q-r_{n} B q\right)\right\|^{2}+\left\|u_{n}-q\right\|^{2}\right. \\
& \left.-\left\|\left(x_{n}-r_{n} B x_{n}\right)-\left(q-r_{n} B q\right)-\left(u_{n}-q\right)\right\|^{2}\right\} \\
\leq & \frac{1}{2}\left\{\left\|x_{n}-q\right\|^{2}+\left\|u_{n}-q\right\|^{2}-\left\|x_{n}-u_{n}-r_{n}\left(B x_{n}-B q\right)\right\|^{2}\right\} \\
\leq & \frac{1}{2}\left\{\left\|x_{n}-q\right\|^{2}+\left\|u_{n}-q\right\|^{2}-\left\|x_{n}-u_{n}\right\|^{2}\right. \\
& \left.+2 r_{n}\left\langle x_{n}-u_{n}, B x_{n}-B q\right\rangle-r_{n}^{2}\left\|B x_{n}-B q\right\|^{2}\right\},
\end{aligned}
$$

which implies that

$$
\left\|u_{n}-q\right\|^{2} \leq\left\|x_{n}-q\right\|^{2}-\left\|x_{n}-u_{n}\right\|^{2}+2 r_{n}\left\|x_{n}-u_{n}\right\|\left\|B x_{n}-B q\right\| .
$$


From (3.1), we get

$$
\begin{aligned}
\left\|y_{n}-x_{n}\right\| & =\left\|P_{C}\left(I-\lambda_{n}(A-\gamma f)\right) x_{n}-P_{C} x_{n}\right\| \\
& \leq\left\|\left(I-\lambda_{n}(A-\gamma f)\right) x_{n}-x_{n}\right\| \\
& \leq \lambda_{n}\left\|(A-\gamma f) x_{n}\right\| .
\end{aligned}
$$

By (C3), we have

$$
\lim _{n \rightarrow \infty}\left\|y_{n}-x_{n}\right\|=0
$$

Setting $w_{n}=\left[I-\lambda_{n}(A-\gamma f)\right] x_{n}$. It follows that

$$
\begin{aligned}
\left\|w_{n}-x_{n}\right\| & =\left\|\left[I-\lambda_{n}(A-\gamma f)\right] x_{n}-x_{n}\right\| \\
& \leq\left\|\left[I-\lambda_{n}(A-\gamma f)\right] x_{n}-x_{n}\right\| \\
& \leq \lambda_{n}\left\|(A-\gamma f) x_{n}\right\| .
\end{aligned}
$$

By using (C3) again, we get

$$
\lim _{n \rightarrow \infty}\left\|w_{n}-x_{n}\right\|=0
$$

From $y_{n}=P_{C}\left[I-\lambda_{n}(A-\gamma f)\right] x_{n}$, we compute

$$
\begin{aligned}
\left\|y_{n}-q\right\| & =\left\|P_{C}\left[I-\lambda_{n}(A-\gamma f)\right] x_{n}-P_{C} q\right\| \\
& \leq\left\|\left[I-\lambda_{n}(A-\gamma f)\right] x_{n}-q\right\| \\
& =\left\|w_{n}-q\right\| .
\end{aligned}
$$

It follows from (3.15) that

$$
\left\|x_{n}-q\right\| \leq\left\|w_{n}-q\right\| .
$$

Then we get

$$
\begin{aligned}
\left\|w_{n}-q\right\|^{2} & \leq\left\langle\left[I-\lambda_{n}(A-\gamma f)\right] x_{n}-q, w_{n}-q\right\rangle \\
& =\lambda_{n}\left\langle\gamma f x_{n}-A q, w_{n}-q\right\rangle+\left\langle\left(I-\lambda_{n} A\right)\left(x_{n}-q\right), w_{n}-q\right\rangle \\
& \leq \lambda_{n}\left\langle\gamma f x_{n}-A q, w_{n}-q\right\rangle+\left(1-\lambda_{n} \bar{\gamma}\right)\left\|x_{n}-q\right\|\left\|w_{n}-q\right\| \\
& \leq\left(1-\lambda_{n} \bar{\gamma}\right)\left\|w_{n}-q\right\|^{2}+\lambda_{n}\left\langle\gamma f x_{n}-A q, w_{n}-q\right\rangle .
\end{aligned}
$$

It follows that

$$
\begin{aligned}
\left\|w_{n}-q\right\|^{2} & \leq \frac{1}{\bar{\gamma}}\left\langle\gamma f x_{n}-A q, w_{n}-q\right\rangle \\
& =\frac{1}{\bar{\gamma}}\left[\gamma\left\langle f x_{n}-f q, w_{n}-q\right\rangle+\left\langle\gamma f q-A q, w_{n}-q\right\rangle\right] \\
& \leq \frac{1}{\bar{\gamma}}\left[\gamma \rho\left\|w_{n}-q\right\|^{2}+\left\langle(A-\gamma f) q, q-w_{n}\right\rangle\right]
\end{aligned}
$$


that is,

$$
\left\|w_{n}-q\right\|^{2} \leq \frac{1}{\bar{\gamma}-\gamma \rho}\left\langle(A-\gamma f) q, q-w_{n}\right\rangle .
$$

On the other hand, we note that

$$
\begin{aligned}
\left\|u_{n}-q\right\|^{2} & =\left\|T_{r_{n}}\left(x_{n}-r_{n} B x_{n}\right)-T_{r_{n}}\left(q-r_{n} B q\right)\right\|^{2} \\
& \leq\left\|\left(x_{n}-r_{n} B x_{n}\right)-\left(q-r_{n} B q\right)\right\|^{2} \\
& =\left\|\left(x_{n}-q\right)-r_{n}\left(B x_{n}-B q\right)\right\|^{2} \\
& \leq\left\|x_{n}-q\right\|^{2}-2 r_{n}\left\langle x_{n}-q, B x_{n}-B q\right\rangle+r_{n}^{2}\left\|B x_{n}-B q\right\|^{2} \\
& \leq\left\|x_{n}-q\right\|^{2}-2 r_{n} \beta\left\|B x_{n}-B q\right\|^{2}+r_{n}^{2}\left\|B x_{n}-B q\right\|^{2} .
\end{aligned}
$$

Using (3.17), (3.18), (3.19), and (3.20), we note that

$$
\begin{aligned}
\left\|x_{n+1}-q\right\|^{2} \leq & \alpha_{n} \beta_{n}\left\|x_{n}-q\right\|^{2}+\alpha_{n}\left(1-\beta_{n}\right)\left\|y_{n}-q\right\|^{2}+\left(1-\alpha_{n}\right)\left\|u_{n}-q\right\|^{2} \\
\leq & \alpha_{n} \beta_{n}\left\|w_{n}-q\right\|^{2}+\alpha_{n}\left(1-\beta_{n}\right)\left\|w_{n}-q\right\|^{2}+\left(1-\alpha_{n}\right)\left\|u_{n}-q\right\|^{2} \\
= & \alpha_{n}\left\|w_{n}-q\right\|^{2}+\left(1-\alpha_{n}\right)\left\|u_{n}-q\right\|^{2} \\
\leq & \frac{\alpha_{n}}{\bar{\gamma}-\gamma \rho}\left\langle(A-\gamma f) q, q-w_{n}\right\rangle \\
& +\left(1-\alpha_{n}\right)\left\{\left\|x_{n}-q\right\|^{2}-2 r_{n} \beta\left\|B x_{n}-B q\right\|^{2}+r_{n}^{2}\left\|B x_{n}-B q\right\|^{2}\right\} \\
= & \frac{\alpha_{n}}{\bar{\gamma}-\gamma \rho}\left\langle(A-\gamma f) q, q-w_{n}\right\rangle \\
& +\left(1-\alpha_{n}\right)\left\{\left\|x_{n}-q\right\|^{2}+r_{n}\left(r_{n}-2 \beta\right)\left\|B x_{n}-B q\right\|^{2}\right\} \\
\leq & \frac{\alpha_{n}}{\bar{\gamma}-\gamma \rho}\left\langle(A-\gamma f) q, q-w_{n}\right\rangle+\left\|x_{n}-q\right\|^{2} \\
& +\left(1-\alpha_{n}\right) r_{n}\left(r_{n}-2 \beta\right)\left\|B x_{n}-B q\right\|^{2} .
\end{aligned}
$$

Then we have

$$
\begin{aligned}
(1 & \left.-\alpha_{n}\right) c(2 \beta-d)\left\|B x_{n}-B q\right\|^{2} \\
& \leq \frac{\alpha_{n}}{\bar{\gamma}-\gamma \rho}\left\langle(A-\gamma f) q, q-w_{n}\right\rangle+\left\|x_{n}-q\right\|^{2}-\left\|x_{n+1}-q\right\|^{2} \\
& \leq \frac{\alpha_{n}}{\bar{\gamma}-\gamma \rho}\left\langle(A-\gamma f) q, q-w_{n}\right\rangle+\left\|x_{n}-x_{n+1}\right\|\left(\left\|x_{n}-q\right\|+\left\|x_{n+1}-q\right\|\right) .
\end{aligned}
$$

From (C3), $\left\{r_{n}\right\} \subset[c, d] \subset(0,2 \beta)$, and (3.12), we obtain

$$
\lim _{n \rightarrow \infty}\left\|B x_{n}-B q\right\|=0
$$

Substituting (3.13) into (3.21), we have

$$
\begin{aligned}
\left\|x_{n+1}-q\right\|^{2} & \leq \alpha_{n}\left\|w_{n}-q\right\|^{2}+\left(1-\alpha_{n}\right)\left\|u_{n}-q\right\|^{2} \\
& \leq \alpha_{n}\left\|w_{n}-q\right\|^{2}+\left(1-\alpha_{n}\right)\left\{\left\|x_{n}-q\right\|^{2}-\left\|x_{n}-u_{n}\right\|^{2}\right.
\end{aligned}
$$




$$
\begin{aligned}
& \left.+2 r_{n}\left\|x_{n}-u_{n}\right\|\left\|B x_{n}-B q\right\|\right\} \\
\leq & \alpha_{n}\left\|w_{n}-q\right\|^{2}+\left\|x_{n}-q\right\|^{2}-\left(1-\alpha_{n}\right)\left\|x_{n}-u_{n}\right\|^{2} \\
& +2 r_{n}\left(1-\alpha_{n}\right)\left\|x_{n}-u_{n}\right\|\left\|B x_{n}-B q\right\|
\end{aligned}
$$

and it follows that

$$
\begin{aligned}
\left(1-\alpha_{n}\right)\left\|x_{n}-u_{n}\right\|^{2} \leq & \alpha_{n}\left\|w_{n}-q\right\|^{2}+\left\|x_{n}-q\right\|^{2}-\left\|x_{n+1}-q\right\|^{2} \\
& +2 r_{n}\left(1-\alpha_{n}\right)\left\|x_{n}-u_{n}\right\|\left\|B x_{n}-B q\right\| \\
\leq & \alpha_{n}\left\|w_{n}-q\right\|^{2}+\left\|x_{n}-x_{n+1}\right\|\left(\left\|x_{n}-q\right\|+\left\|x_{n+1}-q\right\|\right) \\
& +2 r_{n}\left(1-\alpha_{n}\right)\left\|x_{n}-u_{n}\right\|\left\|B x_{n}-B q\right\| .
\end{aligned}
$$

Since we have (C3), (3.12), and (3.22),

$$
\lim _{n \rightarrow \infty}\left\|x_{n}-u_{n}\right\|=0
$$

By (C4), we obtain

$$
\lim _{n \rightarrow \infty}\left\|\frac{x_{n}-u_{n}}{r_{n}}\right\|=\lim _{n \rightarrow \infty} \frac{1}{r_{n}}\left\|x_{n}-u_{n}\right\|=0
$$

Step 4. Next, we will show that

$$
\limsup _{n \rightarrow \infty}\left\langle(\gamma f-A) x^{*}, x_{n}-x^{*}\right\rangle \leq 0
$$

Indeed, we choose a subsequence $\left\{x_{n_{i}}\right\}$ of $\left\{x_{n}\right\}$ such that

$$
\limsup _{n \rightarrow \infty}\left\langle(\gamma f-A) x^{*}, x_{n}-x^{*}\right\rangle=\lim _{i \rightarrow \infty}\left\langle(\gamma f-A) x^{*}, x_{n_{i}}-x^{*}\right\rangle .
$$

Since $\left\{x_{n_{i}}\right\}$ is bounded, there exists a subsequence $\left\{x_{n_{i_{j}}}\right\}$ of $\left\{x_{n_{i}}\right\}$ which converges weakly to $z \in C$. We notice that $\left\|w_{n}-x_{n}\right\| \leq \lambda_{n}\left\|(A-\gamma f) x_{n}\right\| \rightarrow 0$. Hence, we get lim $\sup _{n \rightarrow \infty}\langle(\gamma f-$ $\left.A) x^{*}, x_{n}-x^{*}\right\rangle \leq 0$. Next, we will show that $z \in \operatorname{GMEP}(F, \varphi, B)$. Since $u_{n}=T_{r_{n}}\left(x_{n}-r_{n} B x_{n}\right)$, we have

$$
F\left(u_{n}, y\right)+\left\langle B x_{n}, y-u_{n}\right\rangle+\varphi(y)-\varphi\left(u_{n}\right)+\frac{1}{r_{n}}\left\langle y-u_{n}, u_{n}-x_{n}\right\rangle \geq 0, \quad \forall y \in C .
$$

From (A2), we also have

$$
\left\langle B x_{n}, y-u_{n}\right\rangle+\varphi(y)-\varphi\left(u_{n}\right)+\frac{1}{r_{n}}\left\langle y-u_{n}, u_{n}-x_{n}\right\rangle \geq F\left(y, u_{n}\right), \quad \forall y \in C,
$$

and hence

$$
\left\langle B x_{n_{i}}, y-u_{n_{i}}\right\rangle+\varphi(y)-\varphi\left(u_{n_{i}}\right)+\left\langle y-u_{n_{i}}, \frac{u_{n_{i}}-x_{n_{i}}}{r_{n_{i}}}\right\rangle \geq F\left(y, u_{n_{i}}\right), \quad \forall y \in C .
$$


For $t$ with $0<t \leq 1$ and $y \in C$, let $y_{t}=t y+(1-t) z$. Since $y \in C$ and $z \in C$, we have $y_{t} \in C$. So, from (3.25), we have

$$
\begin{aligned}
\left\langle y_{t}-u_{n_{i}}, B y_{t}\right\rangle \geq & \left\langle y_{t}-u_{n_{i}}, B y_{t}\right\rangle-\varphi\left(y_{t}\right)+\varphi\left(u_{n_{i}}\right)-\left\langle y_{t}-u_{n_{i}}, B x_{n_{i}}\right\rangle \\
& -\left\langle y_{t}-u_{n_{i}}, \frac{u_{n_{i}}-x_{n_{i}}}{r_{n_{i}}}\right\rangle+F\left(y_{t}, u_{n_{i}}\right) \\
= & \left\langle y_{t}-u_{n_{i}}, B y_{t}-B u_{n_{i}}\right\rangle+\left\langle y_{t}-u_{n_{i}}, B u_{n_{i}}-B x_{n_{i}}\right\rangle-\varphi\left(y_{t}\right)+\varphi\left(u_{n_{i}}\right) \\
& -\left\langle y_{t}-u_{n_{i}}, \frac{u_{n_{i}}-x_{n_{i}}}{r_{n_{i}}}\right\rangle+F\left(y_{t}, u_{n_{i}}\right) .
\end{aligned}
$$

Since $\left\|u_{n_{i}}-x_{n_{i}}\right\| \rightarrow 0$, we have $\left\|B u_{n_{i}}-B x_{n_{i}}\right\| \rightarrow 0$. Further, from the inverse strongly monotonicity of $B$, we have $\left\langle y_{t}-u_{n_{i}}, B y_{t}-B u_{n_{i}}\right\rangle \geq 0$. So, from (A4), (A5), and the weak lower semicontinuity of $\varphi, \frac{u_{n_{i}}-x_{n_{i}}}{r_{n_{i}}} \rightarrow 0$ and $u_{n_{i}} \rightarrow w$, we have in the limit

$$
\left\langle y_{t}-w, B y_{t}\right\rangle \geq-\varphi\left(y_{t}\right)+\varphi(w)+F\left(y_{t}, w\right)
$$

as $i \rightarrow \infty$. From (A1), (A4) and (3.26), we also get

$$
\begin{aligned}
0 & =F\left(y_{t}, y_{t}\right)+\varphi\left(y_{t}\right)-\varphi\left(y_{t}\right) \\
& \leq t F\left(y_{t}, y\right)+(1-t) F\left(y_{t}, z\right)+t \varphi(y)-(1-t) \varphi(z)-\varphi\left(y_{t}\right) \\
& =t\left[F\left(y_{t}, y\right)+\varphi(y)-\varphi\left(y_{t}\right)\right]+(1-t)\left[F\left(y_{t}, z\right)+\varphi(z)-\varphi\left(y_{t}\right)\right] \\
& \leq t\left[F\left(y_{t}, y\right)+\varphi(y)-\varphi\left(y_{t}\right)\right]+(1-t)\left\langle y_{t}-z, B y_{t}\right\rangle \\
& =t\left[F\left(y_{t}, y\right)+\varphi(y)-\varphi\left(y_{t}\right)\right]+(1-t) t\left\langle y-z, B y_{t}\right\rangle, \\
0 & \leq F\left(y_{t}, y\right)+\varphi(y)-\varphi\left(y_{t}\right)+(1-t)\left\langle y-z, B y_{t}\right\rangle .
\end{aligned}
$$

Letting $t \rightarrow 0$, we have, for each $y \in C$,

$$
F(z, y)+\varphi(y)-\varphi(z)+\langle y-z, B z\rangle \geq 0
$$

This implies that $z \in \operatorname{GMEP}(F, \varphi, B)$. It is easy to see that $P_{G M E P(F, \varphi, B)}(I-A+\gamma f)\left(x^{*}\right)$ is a contraction of $H$ into itself. Hence $H$ is complete, there exists a unique fixed point $x^{*} \in H$, such that $x^{*}=P_{G M E P(F, \varphi, B)}(I-A+\gamma f)\left(x^{*}\right)$.

Step 5. Next, we will prove $x_{n} \rightarrow x^{*} \in \operatorname{GMEP}(F, \varphi, B)$, which solves the variational inequality (1.8). It follows from (3.1) that

$$
\begin{aligned}
\left\|x_{n+1}-x^{*}\right\|^{2}= & \alpha_{n} \beta_{n}\left\langle x_{n}-x^{*}, x_{n+1}-x^{*}\right\rangle \\
& +\alpha_{n}\left(1-\beta_{n}\right)\left\langle P_{C}\left[I-\lambda_{n}(A-\gamma f)\right] x_{n}-P_{C}\left[I-\lambda_{n}(A-\gamma f)\right] x^{*}, x_{n+1}-x^{*}\right\rangle \\
& +\alpha_{n}\left(1-\beta_{n}\right)\left\langle P_{C}\left[I-\lambda_{n}(A-\gamma f)\right] x^{*}-x^{*}, x_{n+1}-x^{*}\right\rangle \\
& +\left(1-\alpha_{n}\right)\left\langle T_{r_{n}}\left(I-r_{n} B\right) x_{n}-T_{r_{n}}\left(I-r_{n} B\right) x^{*}, x_{n+1}-x^{*}\right\rangle \\
\leq & \alpha_{n} \beta_{n}\left\|x_{n}-x^{*}\right\|\left\|x_{n+1}-x^{*}\right\| \\
& +\alpha_{n}\left(1-\beta_{n}\right)\left\langle\left[I-\lambda_{n}(A-\gamma f)\right] x_{n}-\left[I-\lambda_{n}(A-\gamma f)\right] x^{*}, x_{n+1}-x^{*}\right\rangle
\end{aligned}
$$




$$
\begin{aligned}
& +\alpha_{n}\left(1-\beta_{n}\right)\left\langle\left[I-\lambda_{n}(A-\gamma f)\right] x^{*}-x^{*}, x_{n+1}-x^{*}\right\rangle \\
& +\left(1-\alpha_{n}\right)\left\|T_{r_{n}}\left(I-r_{n} B\right) x_{n}-T_{r_{n}}\left(I-r_{n} B\right) x^{*}\right\|\left\|x_{n+1}-x^{*}\right\| \\
& \leq \alpha_{n} \beta_{n}\left\|x_{n}-x^{*}\right\|\left\|x_{n+1}-x^{*}\right\| \\
& +\alpha_{n}\left(1-\beta_{n}\right)\left\{\left[1-(\bar{\gamma}-\gamma \rho) \lambda_{n}\right]\left\|x_{n}-x^{*}\right\|\right. \\
& \left.+\lambda_{n}\left\|\gamma f\left(x^{*}\right)-A x^{*}\right\|\right\}\left\|x_{n+1}-x^{*}\right\| \\
& -\alpha_{n}\left(1-\beta_{n}\right) \lambda_{n}\left\langle(A-\gamma f) x^{*}, x_{n+1}-x^{*}\right\rangle+\left(1-\alpha_{n}\right)\left\|x_{n}-x^{*}\right\|\left\|x_{n+1}-x^{*}\right\| \\
& =\alpha_{n} \beta_{n}\left\|x_{n}-x^{*}\right\|\left\|x_{n+1}-x^{*}\right\|+\left(1-\alpha_{n}\right)\left\|x_{n}-x^{*}\right\|\left\|x_{n+1}-x^{*}\right\| \\
& +\alpha_{n}\left(1-\beta_{n}\right)\left[1-(\bar{\gamma}-\gamma \rho) \lambda_{n}\right]\left\|x_{n}-x^{*}\right\|\left\|x_{n+1}-x^{*}\right\| \\
& +\alpha_{n}\left(1-\beta_{n}\right) \lambda_{n}\left\|\gamma f\left(x^{*}\right)-A x^{*}\right\|\left\|x_{n+1}-x^{*}\right\| \\
& -\alpha_{n}\left(1-\beta_{n}\right) \lambda_{n}\left|(A-\gamma f) x^{*}, x_{n+1}-x^{*}\right\rangle \\
& =\left[1-\alpha_{n}\left(1-\beta_{n}\right)\right]\left\|x_{n}-x^{*}\right\|\left\|x_{n+1}-x^{*}\right\| \\
& +\alpha_{n}\left(1-\beta_{n}\right)\left[1-(\bar{\gamma}-\gamma \rho) \lambda_{n}\right]\left\|x_{n}-x^{*}\right\|\left\|x_{n+1}-x^{*}\right\| \\
& +\alpha_{n}\left(1-\beta_{n}\right) \lambda_{n}\left\|\gamma f\left(x^{*}\right)-A x^{*}\right\|\left\|x_{n+1}-x^{*}\right\| \\
& -\alpha_{n}\left(1-\beta_{n}\right) \lambda_{n}\left|(A-\gamma f) x^{*}, x_{n+1}-x^{*}\right\rangle \\
& =\left[1-\alpha_{n}\left(1-\beta_{n}\right)\left[1-1+(\bar{\gamma}-\gamma \rho) \lambda_{n}\right]\right]\left\|x_{n}-x^{*}\right\|\left\|x_{n+1}-x^{*}\right\| \\
& +\alpha_{n}\left(1-\beta_{n}\right) \lambda_{n}\left\|\gamma f\left(x^{*}\right)-A x^{*}\right\|\left\|x_{n+1}-x^{*}\right\| \\
& -\alpha_{n}\left(1-\beta_{n}\right) \lambda_{n}\left|(A-\gamma f) x^{*}, x_{n+1}-x^{*}\right\rangle \\
& =\left[1-\alpha_{n}\left(1-\beta_{n}\right)(\bar{\gamma}-\gamma \rho) \lambda_{n}\right]\left\|x_{n}-x^{*}\right\|\left\|x_{n+1}-x^{*}\right\| \\
& +\alpha_{n}\left(1-\beta_{n}\right) \lambda_{n}\left\|\gamma f\left(x^{*}\right)-A x^{*}\right\|\left\|x_{n+1}-x^{*}\right\| \\
& -\alpha_{n}\left(1-\beta_{n}\right) \lambda_{n}\left|(A-\gamma f) x^{*}, x_{n+1}-x^{*}\right\rangle \\
& \leq \frac{1-\alpha_{n}\left(1-\beta_{n}\right)(\bar{\gamma}-\gamma \rho) \lambda_{n}}{2}\left(\left\|x_{n}-x^{*}\right\|^{2}+\left\|x_{n+1}-x^{*}\right\|^{2}\right) \\
& +\alpha_{n}\left(1-\beta_{n}\right) \lambda_{n}\left\|\gamma f\left(x^{*}\right)-A x^{*}\right\|\left\|x_{n+1}-x^{*}\right\| \\
& -\alpha_{n}\left(1-\beta_{n}\right) \lambda_{n}\left\langle(A-\gamma f) x^{*}, x_{n+1}-x^{*}\right\rangle \\
& \leq \frac{1-\left(1-\beta_{n}\right)(\bar{\gamma}-\gamma \rho) \alpha_{n} \lambda_{n}}{2}\left\|x_{n}-x^{*}\right\|^{2}+\frac{1}{2}\left\|x_{n+1}-x^{*}\right\|^{2} \\
& +\left(1-\beta_{n}\right) \alpha_{n} \lambda_{n}\left\|\gamma f\left(x^{*}\right)-A x^{*}\right\|\left\|x_{n+1}-x^{*}\right\| \\
& -\left(1-\beta_{n}\right) \alpha_{n} \lambda_{n}\left\langle(A-\gamma f) x^{*}, x_{n+1}-x^{*}\right\rangle \text {, }
\end{aligned}
$$

which implies that

$$
\begin{aligned}
\left\|x_{n+1}-x^{*}\right\|^{2} \leq & {\left[1-\left(1-\beta_{n}\right)(\bar{\gamma}-\gamma \rho) \alpha_{n} \lambda_{n}\right]\left\|x_{n}-x^{*}\right\|^{2} } \\
& +2\left(1-\beta_{n}\right) \alpha_{n} \lambda_{n}\left\|\gamma f\left(x^{*}\right)-A x^{*}\right\|\left\|x_{n+1}-x^{*}\right\| \\
& -2\left(1-\beta_{n}\right) \alpha_{n} \lambda_{n}\left\langle(A-\gamma f) x^{*}, x_{n+1}-x^{*}\right\rangle .
\end{aligned}
$$


Since $\left\{x_{n}\right\},\left\{f\left(x_{n}\right)\right\}$, and $\left\{A x_{n}\right\}$ are all bounded, we can choose a constant $M>0$ such that

$$
\sup \frac{1}{\bar{\gamma}-\gamma \rho}\left\{2\left\|\gamma f\left(x^{*}\right)-A x^{*}\right\|\left\|x_{n+1}-x^{*}\right\|+2\left\langle(A-\gamma f) x^{*}, x_{n+1}-x^{*}\right)\right\} \leq M .
$$

It follows that

$$
\left\|x_{n+1}-x^{*}\right\|^{2} \leq\left[1-\left(1-\beta_{n}\right)(\bar{\gamma}-\gamma \rho) \alpha_{n} \lambda_{n}\right]\left\|x_{n}-x^{*}\right\|^{2}+\left(1-\beta_{n}\right) \alpha_{n} \lambda_{n} M
$$

By (C3), we conclude that $x_{n} \rightarrow x^{*}$, as $n \rightarrow \infty$. This completes the proof.

\section{An example}

Next, the following example shows that all conditions of Theorem 3.1 are satisfied.

Example 4.1 For instance, let $\alpha_{n}=\frac{n+1}{n^{2}+1}, \beta_{n}=\frac{1}{n+1}, \lambda_{n}=\frac{1}{2(n+1)}$, and $r_{n}=\frac{n}{n+1}$. Then clearly the sequences $\left\{\alpha_{n}\right\},\left\{\lambda_{n}\right\}$ satisfy the following condition:

$$
\frac{n+1}{n^{2}+1} \leq \frac{1}{2(n+1)}
$$

We will show that the condition $(\mathrm{C} 1)$ is fulfilled. Indeed, we have

$$
\begin{aligned}
\sum_{n=1}^{\infty}\left|\alpha_{n}-\alpha_{n-1}\right| & =\sum_{n=1}^{\infty}\left|\frac{n+1}{n^{2}+1}-\frac{n}{(n-1)^{2}+1}\right| \\
& =\sum_{n=1}^{\infty}\left|\frac{(n+1)\left(n^{2}-2 n+2\right)-n\left(n^{2}+1\right)}{\left(n^{2}+1\right)\left(n^{2}-2 n+2\right)}\right| \\
& =\sum_{n=1}^{\infty}\left|\frac{2+n-n^{2}}{n^{4}-2 n^{3}+3 n^{2}-2 n+2}\right|
\end{aligned}
$$

The sequence $\left\{\alpha_{n}\right\}$ satisfies the condition (C1) by a $p$-series.

Next, we will show that the condition (C2) is fulfilled. We compute

$$
\begin{aligned}
\sum_{n=1}^{\infty}\left|\beta_{n}-\beta_{n-1}\right| & =\sum_{n=1}^{\infty}\left|\frac{1}{n+1}-\frac{1}{n}\right| \\
& =\left(\frac{1}{1}-\frac{1}{2}\right)+\left(\frac{1}{2}-\frac{1}{3}\right)+\left(\frac{1}{3}-\frac{1}{4}\right)+\cdots \\
& =1 .
\end{aligned}
$$

The sequence $\left\{\beta_{n}\right\}$ satisfies the condition (C2).

Next, we will show that the condition (C3) is fulfilled. We compute

$$
\begin{aligned}
\sum_{n=1}^{\infty}\left|\lambda_{n}-\lambda_{n-1}\right| & =\sum_{n=1}^{\infty}\left|\frac{1}{2(n+1)}-\frac{1}{2 n}\right| \\
& =\left(\frac{1}{2 \cdot 1}-\frac{1}{2 \cdot 2}\right)+\left(\frac{1}{2 \cdot 2}-\frac{1}{2 \cdot 3}\right)+\left(\frac{1}{2 \cdot 3}-\frac{1}{2 \cdot 4}\right)+\cdots \\
& =\frac{1}{2}
\end{aligned}
$$




$$
\lim _{n \rightarrow \infty} \lambda_{n}=\lim _{n \rightarrow \infty} \frac{1}{2(n+1)}=0,
$$

and

$$
\sum_{n=1}^{\infty} \lambda_{n}=\sum_{n=1}^{\infty} \frac{1}{2(n+1)}=\infty .
$$

The sequence $\left\{\lambda_{n}\right\}$ satisfies the condition (C3).

Finally, we will show that the condition (C4) is fulfilled. We compute

$$
\begin{aligned}
\sum_{n=1}^{\infty}\left|r_{n}-r_{n-1}\right| & =\sum_{n=1}^{\infty}\left|\frac{n}{n+1}-\frac{n-1}{(n-1)+1}\right| \\
& =\sum_{n=1}^{\infty}\left|\frac{n(n)-(n-1)(n+1)}{(n+1) n}\right| \\
& =\sum_{n=1}^{\infty}\left|\frac{n^{2}-n^{2}+1}{(n+1) n}\right| \\
& =\sum_{n=1}^{\infty}\left|\frac{1}{n(n+1)}\right|
\end{aligned}
$$

and

$$
\liminf _{n \rightarrow \infty} r_{n}=\liminf _{n \rightarrow \infty} \frac{n}{n+1}=1
$$

The sequence $\left\{r_{n}\right\}$ satisfies the condition (C4).

Corollary 4.2 Let $C$ be a nonempty closed convex subset of a real Hilbert space H. Let $A: H \rightarrow H$ be a strongly positive linear bounded operator, $f: C \rightarrow H$ be $\rho$-contraction, $\gamma$ be a positive real number such that $\frac{\bar{\gamma}-1}{\rho}<\gamma<\frac{\bar{\gamma}}{\rho}$ and $T: C \rightarrow C$ be a nonexpansive mapping with $F(T) \neq \emptyset$. Let $\left\{x_{n}\right\}$ be a sequence generated by the following algorithm for arbitrary $x_{0} \in C$ :

$$
x_{n+1}=\beta_{n} x_{n}+\left(1-\beta_{n}\right) T P_{C}\left[I-\lambda_{n}(A-\gamma f)\right] x_{n},
$$

where $\left\{\beta_{n}\right\},\left\{\lambda_{n}\right\} \subset[0,1]$ satisfy the following conditions:

(C1) $\sum_{n=1}^{\infty}\left|\beta_{n+1}-\beta_{n}\right|<\infty, 0<\liminf _{n \rightarrow \infty} \beta_{n}<\limsup _{n \rightarrow \infty} \beta_{n}<1$;

(C2) $\sum_{n=1}^{\infty}\left|\lambda_{n+1}-\lambda_{n}\right|<\infty, \sum_{n=1}^{\infty} \lambda_{n}=\infty, \lim _{n \rightarrow \infty} \lambda_{n}=0$.

Then $\left\{x_{n}\right\}$ converges strongly to $x^{*} \in F(T)$, which is the unique solution of the variational inequality:

$$
\left\langle(A-\gamma f) x^{*}, x-x^{*}\right\rangle \geq 0, \quad \forall x \in F(T) .
$$

Proof Setting $\left\{\alpha_{n}\right\} \equiv 1$ and $T$ to be a nonexpansive mapping in Theorem 3.1, we obtain the desired conclusion immediately.

Remark 4.3 Corollary 4.2 generalizes and improves the results of Yao et al. [11]. 
Corollary 4.4 Let $C$ be a nonempty closed convex subset of a real Hilbert space H. Let A : $H \rightarrow H$ be a strongly positive linear bounded operator, $B: C \rightarrow H$ be a $\beta$-inverse-strongly monotone and $F$ be a bifunction from $C \times C \rightarrow \mathcal{R}$ satisfying (A1)-(A5) and let $\varphi: C \rightarrow \mathcal{R}$ be convex and lower semicontinuous with either (B1) or (B2). Suppose $\operatorname{GMEP}(F, \varphi, B) \neq \emptyset$. Let $\left\{x_{n}\right\}$ be a sequence by the following algorithm for arbitrary $x_{0} \in C$ :

$$
x_{n+1}=\alpha_{n}\left(\beta_{n} x_{n}+\left(1-\beta_{n}\right)\left[I-\lambda_{n} A\right] x_{n}\right)+\left(1-\alpha_{n}\right) T_{r_{n}}\left(I-r_{n} B\right) x_{n},
$$

where $\left\{\alpha_{n}\right\},\left\{\beta_{n}\right\},\left\{\lambda_{n}\right\} \subset[0,1], \alpha_{n} \leq \lambda_{n}$, and $r_{n} \in(0,2 \beta)$ satisfy the following conditions:

(C1) $\sum_{n=1}^{\infty}\left|\alpha_{n}-\alpha_{n-1}\right|<\infty$;

(C2) $\sum_{n=1}^{\infty}\left|\beta_{n}-\beta_{n-1}\right|<\infty$;

(C3) $\sum_{n=1}^{\infty}\left|\lambda_{n}-\lambda_{n-1}\right|<\infty, \sum_{n=1}^{\infty} \lambda_{n}=\infty, \lim _{n \rightarrow \infty} \lambda_{n}=0$;

(C4) $\sum_{n=1}^{\infty}\left|r_{n}-r_{n-1}\right|<\infty, \liminf _{n \rightarrow \infty} r_{n}>0$.

Then $\left\{x_{n}\right\}$ converges strongly to $x^{*} \in \operatorname{GMEP}(F, \varphi, B)$, which is the unique solution of the variational inequality:

$$
\left\langle A x^{*}, x-x^{*}\right\rangle \geq 0, \quad \forall x \in \operatorname{GMEP}(F, \varphi, B) .
$$

Proof Setting $T, P_{C}$ to be the identity and $f \equiv 0$ in Theorem 3.1, we obtain the desired conclusion immediately.

Corollary 4.5 Let $C$ be a nonempty closed convex subset of a real Hilbert space $H$. Let $A: H \rightarrow H$ be a strongly positive linear bounded operator, $f: C \rightarrow H$ be $\rho$-contraction, $B:$ $C \rightarrow H$ be $\beta$-inverse-strongly monotone and $F$ be a bifunction from $C \times C \rightarrow \mathcal{R}$ satisfying (A1)-(A5) and let $\varphi: C \rightarrow \mathcal{R}$ be convex and lower semicontinuous with either (B1) or (B2). Let $\left\{x_{n}\right\}$ be a sequence generated by the following algorithm for arbitrary $x_{0} \in C$ :

$$
x_{n+1}=\alpha_{n}\left(\lambda_{n}\left(1-\beta_{n}\right) f\left(x_{n}\right)+\left[I-\lambda_{n}\left(1-\beta_{n}\right) A\right] x_{n}\right)+\left(1-\alpha_{n}\right) T_{r_{n}}\left(I-r_{n} B\right) x_{n},
$$

where $\left\{\alpha_{n}\right\},\left\{\beta_{n}\right\},\left\{\lambda_{n}\right\} \subset[0,1], \lambda_{n} \leq \beta_{n}$, and $r_{n} \in(0,2 \beta)$ satisfy the following conditions:

(C1) $\sum_{n=1}^{\infty}\left|\alpha_{n+1}-\alpha_{n}\right|<\infty$;

(C2) $\sum_{n=1}^{\infty}\left|\beta_{n+1}-\beta_{n}\right|<\infty$;

(C3) $\sum_{n=1}^{\infty}\left|\lambda_{n+1}-\lambda_{n}\right|<\infty, \sum_{n=1}^{\infty} \lambda_{n}=\infty, \lim _{n \rightarrow \infty} \lambda_{n}=0$;

(C4) $\sum_{n=1}^{\infty}\left|r_{n+1}-r_{n}\right|<\infty, \liminf _{n \rightarrow \infty} r_{n}>0$.

Then $\left\{x_{n}\right\}$ converges strongly to $x^{*} \in \operatorname{GMEP}(F, \varphi, B)$, which is the unique solution of the variational inequality

$$
\left\langle(A-f) x^{*}, x-x^{*}\right\rangle \geq 0, \quad \forall x \in G M E P(F, \varphi, B) .
$$

Proof Setting $T, P_{C}$ to be the identity and $\gamma \equiv 1$ in Theorem 3.1, we obtain the desired conclusion immediately.

The authors declare that they have no competing interests. 


\section{Author details}

${ }^{1}$ Department of Mathematics, Faculty of Science, King Mongkut's University of Technology Thonburi, 126 Pracha u-thit Rd., Bang Mod, Thrung Khru, Bangmod, Bangkok, 10140, Thailand. ${ }^{2}$ Centre of Excellence in Mathematics, CHE, Si Ayutthaya Road, Bangkok, 10400, Thailand. ${ }^{3}$ Department of Mathematics, Faculty of Science and Agriculture, Rajamangala University of Technology Lanna, Phan, Chiangrai, 57120, Thailand. ${ }^{4}$ Department of Logistic Engineering, Faculty of Engineering, Rajamangala University of Technology Lanna, Phan, Chiangrai, 57120, Thailand.

\section{Acknowledgements}

This project was partially supported by Centre of Excellence in Mathematics, the Commission on Higher Education, Ministry of Education, Thailand. The second author and third author were supported by Innovation park, RMUTL Hands-on Researcher Project, Rajamangala University of Technology Lanna Chiangrai under Grant no. 57HRG-10 during the preparation of this paper

Received: 28 April 2014 Accepted: 5 September 2014 Published: 16 Oct 2014

\section{References}

1. Al-Mazeooei, AE, Latif, A, Yao, JC: Solving generalized mixed equilibria, variational inequalities, and constrained convex minimization. Abstr. Appl. Anal. 2014, Article ID 587865 (2014)

2. Ceng, LC, Chen, CM, Pang, CT: Hybrid extragradient-like viscosity methods for generalized mixed equilibrium problems, variational inclusions, and optimization problems. Abstr. Appl. Anal. 2014, Article ID 120172 (2014)

3. Ceng, LC, Chen, CM, Wen, CF, Pang, CT: Relaxed iterative algorithms for generalized mixed equilibrium problems with constraints of variational inequalities and variational inclusions. Abstr. Appl. Anal. 2014, Article ID 345212 (2014)

4. Ceng, LC, Ho, JL: Hybrid extragradient method with regularization for convex minimization, generalized mixed equilibrium, variational inequality and fixed point problems. Abstr. Appl. Anal. 2014, Article ID 436069 (2014)

5. Blum, E, Oettli, W: From optimization and variational inequalities to equilibrium problems. Math. Stud. 63, 123-145 (1994)

6. Hartman, P, Stampacchia, G: On some nonlinear elliptic differential functional equations. Acta Math. 115, 271-310 (1966)

7. Kirk, WA: Fixed point theorem for mappings which do not increase distance. Am. Math. Mon. 72, 1004-1006 (1965)

8. Yao, YH, Cho, YJ, Yang, PX: An iterative algorithm for a hierarchical problem. J. Appl. Math. 2012, Article ID 320421 (2012)

9. Yao, YH, Kang, Jl, Cho, YJ, Liou, YC: Composite schemes for variational inequalities over equilibrium problems and variational inclusions. J. Inequal. Appl. 2013, Article ID 414 (2013)

10. Yao, Y, Liou, Y-C, Chen, C-P: Hierarchical convergence of a double-net algorithm for equilibrium problems and variational inequality problems. Fixed Point Theory Appl. 2010, Article ID 642584 (2010). doi:10.1155/2010/642584

11. Yao, Y, Liou, Y-C, Kang, SM: Algorithms construction for variational inequalities. Fixed Point Theory Appl. 2011, Article ID 794203 (2011). doi:10.1155/2011/794203

12. Opial, Z: Weak convergence of the sequence of successive approximations for nonexpansive mappings. Bull. Am. Math. Soc. 73, 595-597 (1967)

13. Peng, JW, Yao, JC: A new hybrid-extragradient method for generalized mixed equilibrium problems and fixed point problems and variational inequality problems. Taiwan. J. Math. 12(6), 1401-1432 (2008)

14. Browder, FE: Nonlinear operators and nonlinear equations of evolution in Banach spaces. Proc. Symp. Pure Math. 18, 78-81 (1976)

15. Marino, G, Xu, HK: A general iterative method for nonexpansive mapping in Hilbert space. J. Math. Anal. Appl. 318 43-52 (2006)

16. Xu, HK: Iterative algorithms for nonlinear operators. J. Lond. Math. Soc. 66, 240-256 (2002)

10.1186/1029-242X-2014-405

Cite this article as: Kumam et al.: An explicit algorithm for solving the optimize hierarchical problems. Journal of Inequalities and Applications 2014, 2014:405

\section{Submit your manuscript to a SpringerOpen ${ }^{\ominus}$ journal and benefit from:}

- Convenient online submission

- Rigorous peer review

- Immediate publication on acceptance

- Open access: articles freely available online

- High visibility within the field

- Retaining the copyright to your article 